Research Article

\title{
Finite-Time Attitude Control for Quadrotor with Input Constraints and Disturbances
}

\author{
Zijun Gao $(D$, Jin Wang, and Yaping Tian \\ School of Information Science and Engineering, Dalian Polytechnic University, Dalian, China \\ Correspondence should be addressed to Zijun Gao; gaozijun_dpu@163.com
}

Received 19 January 2020; Revised 19 April 2020; Accepted 25 May 2020; Published 29 June 2020

Academic Editor: Mariano Torrisi

Copyright (c) 2020 Zijun Gao et al. This is an open access article distributed under the Creative Commons Attribution License, which permits unrestricted use, distribution, and reproduction in any medium, provided the original work is properly cited.

\begin{abstract}
This paper investigates the adaptive output feedback attitude control of a quadrotor. First, a nonsingular terminal sliding-mode variable and auxiliary variable are introduced into a closed-loop structure. Meanwhile, a fuzzy logic system is incorporated into an adaptive algorithm to compensate for the adverse influence caused by lumped disturbances including system uncertainty and external disturbances on the attitude adjustment performance of a quadrotor. Then, a novel finite-time output feedback controller equipped with the saturation suppression algorithm is designed. Rigorous proof shows that the design control strategy ensures the closed-loop system stability and guarantees the attitude of the spacecraft to track desired command signals in finite time. Simulation results are presented to illustrate the performance of the proposed control scheme.
\end{abstract}

\section{Introduction}

The challenges faced in the control of a quadrotor with excellent capability have been concerned and developed widely in the last several decades based on the demand for a reliable one for both commercial and military applications. During the quadrotor entry process, the attitude adjustment of the quadrotor involves attitude maneuvering through a wide range of physical limitation, lumped external disturbances, and system parameter uncertainties [1-3]. Many advanced flight control methods have been proposed for the quadrotor's attitude control in order to improve the performance of the control system, such as adaptive backstepping method $[4,5]$, adaptive compensation control $[6-8]$, sliding-mode control strategy $[9,10]$, and linearization control technique $[11,12]$.

Although the high accuracy of attitude adjustment can be accomplished by using the previously discussed control strategy, these works require infinite time to achieve attitude maneuver. It is obvious that the convergence rate with infinite settling time is not ideal during critical phases of some kind of specialized real-time tasks. There can be no doubt that the finite-time control technology $[13,14]$ can provide higher tracking accuracy, faster dynamic response, and better robustness than the asymptotic control approach. Therefore, the finite-time control technique is one of the most effective control strategies that can handle the tracking control task and the attitude stabilization problem. In [15], a time-specified control strategy based on the nonsingular terminal sliding-mode technique was designed to solve the problem of trajectory tracking for robotic airships. In [16], a finite-time control method with time-delay estimation is proposed for shape memory alloy actuators. In [17], an adaptation supertwisting algorithm control scheme using the finite-time technique was designed for attitude tracking of the quadrotor. In [18], a smooth variable structure control scheme with multivariable form and disturbance observer was designed for quadrotor attitude adjustment. A nonsingular terminal sliding-mode control (NTSMC) method using the finite-time technique was thoroughly examined in [19] to achieve attitude tracking maneuver for the quadrotor with atmospheric disturbances. In a more recent work [20], an output feedback attitude controller based on the finitetime strategy was developed for the quadrotor with both matched and mismatched disturbances. Although many finite-time control strategies are focused on how to deal with attitude maneuver of the quadrotor, there is still a challenging problem for improving both the fast response ability 
and robustness of the closed-loop system with the factor of numerous disadvantages in different working environments, which is one motivation of this paper.

Another problem to be noted is actuator saturation, which is generally encountered for nonsmooth nonlinear constraints of actuators in quadrotor applications. In fact, if an actuator falls into its physical saturation limitation boundary, any operation to enlarge the output of the actuator would not make any variation in the system output. It is likely to cause control performance degradation and even leading to task failure due to actuator saturation when the designed controller is not equipped with an effectiveness adjustment strategy to dump the saturated actuators [21-26]. Besides, taking quadrotor parameter uncertainties and large external disturbances into consideration makes the attitude tracking control problem of the quadrotor more difficult. How to cope with the simultaneous action of the abovementioned adverse factors has been turned into a challenging subject, and extensive attentions have been paid to solve the issue of saturation nonlinearity. To the best knowledge of the authors because of the complex characteristics of the quadrotor system, there are few results for the case where a finite-time control method is designed to achieve attitude adjustment of the quadrotor with control torque limitation and lumped disturbances.

In order to respond to the above discussions, we will investigate the adaptive output feedback attitude control based on the finite-time control technique for quadrotor systems with lumped disturbance and input saturation in this paper. In particular, we are interested in developing an effective adaptation compensation algorithm capable of accommodating the lumped disturbances as well as input saturation suppression. The main contributions are summarized as follows:

(1) A control input auxiliary variable is designed into the design of the closed-loop system, and the design parameter update laws are able to make the control input boundedness by the saturation suppression algorithm only.

(2) A fuzzy logic system is incorporated into the parameter updating algorithm to estimate the complex nonlinear function including system uncertainty and external disturbances. Under the design NTSMC structure, a novel compensation control law equipped with the adaptive algorithm is designed to achieve both terminal surface variable stabilization and attitude tracking.

(3) A sliding-mode differentiator is utilized to relax the differential operation requirement of the virtual control law, which avoids the related singular problems. Moreover, the introduced differentiator can achieve virtual law approximation with lower computational complexity, facilitating the control law design.

The paper is organized as follows. In Section 2, the system model with adverse factors is formulated. The closedloop system structure is established by the nonsingular terminal surface and the control input auxiliary variable, and the finite-time stability of the design control strategy with the parameters update law is analyzed in Section 3. Illustrative simulation of the design control strategy for the quadrotor and conclusion is given in Sections 4 and 5, respectively.

\section{Problem Formulation}

2.1. System Model. The quadrotor system model contains the rotational equations and the translational equations of motion. This paper will focus on the attitude controller design for the quadrotor, and therefore a control-oriented model can be expressed as [5-8]

$$
\mathrm{J} \ddot{\Theta}+\mathbf{N} \dot{\Theta}=\mathbf{M}+\mathbf{d},
$$

where $\Theta=[\alpha, \beta, \sigma]^{T} \in \mathbb{R}^{3}$ is the attitude angle vector including the yaw angle $\alpha$, pitch angle $\beta$, and roll angle $\sigma ; \dot{\Theta}$ and $\ddot{\Theta}$ are the attitude angular rate vector and the angular acceleration vector, respectively; $\mathbf{d}=\left[d_{1}, d_{2}, d_{3}\right]^{T}$ is the external disturbance; $\mathbf{M}=\left[M_{1}, M_{2}, M_{3}\right] \in \mathbb{R}^{3}$ is control torque vector subject to nonlinear saturation described as

$$
M_{i}=\left\{\begin{array}{ll}
\operatorname{sign}\left(u_{i}\right) \cdot u_{M}, & \text { if }\left|u_{i}\right| \geq u_{M}, \\
u_{i}, & \text { if }\left|u_{i}\right|<u_{M},
\end{array} \quad(i=1,2,3),\right.
$$

where $u_{M}$ is a known bound of $M_{i}$ and $u_{i}$ is the design control command. In addition, $\mathbf{J} \in \mathbb{R}^{3 \times 3}$ is a symmetric and positive definite matrix, and the matrix $\mathbf{N} \in \mathbb{R}^{3 \times 3}$ is the Coriolis term of the quadrotor containing centrifugal and gyroscope terms ( $\mathbf{J}$ and $\mathbf{N}$ will be given later).

It is worth noting that the expression of $M_{i}$ in (2) is a discontinuous function which will lead to the sharp corner. In order to improve the smoothness of the control input, the saturation nonlinear function $M_{i}$ is approximated by a smooth function defined as

$$
\left\{\begin{array}{l}
\Gamma_{\mathbf{u}}\left(u_{i}\right)=u_{M} \cdot \tanh \left(\frac{u_{i}}{u_{M}}\right) \\
\Gamma_{\mathbf{u}}=\left[\Gamma_{\mathbf{u}}\left(u_{1}\right), \Gamma_{\mathbf{u}}\left(u_{2}\right), \Gamma_{\mathbf{u}}\left(u_{3}\right)\right]^{T}
\end{array}\right.
$$

In light of (3), (2) can be expressed as

$$
M_{i}=\Gamma_{\mathbf{u}}\left(u_{i}\right)+\Delta M_{i}, \quad i=1,2,3,
$$

where $\left|\Delta M_{i}\right|=\left|M_{i}-\Gamma_{\mathbf{u}}\left(u_{i}\right)\right| \leq u_{M}(1-\tanh (1))$ is a bounded approximation error. Furthermore, we have

$$
\mathbf{M}=\Gamma_{\mathbf{u}}+\Delta \mathbf{M},
$$

where $\Delta \mathbf{M}=\left[\Delta M_{1}, \Delta M_{2}, \Delta M_{3}\right]^{T}$, satisfying $\|\Delta \mathbf{M}\|=\sum_{i=1}^{3}$ $\left|\Delta M_{i}\right| \leq 3 \cdot u_{M}(1-\tanh (1))$. Let $\dot{\Theta}=\omega$, then (1) can be rewritten as

$$
\left\{\begin{array}{l}
\dot{\Theta}=\mathbf{\omega} \\
\mathbf{J} \dot{\omega}=-\mathbf{N} \mathbf{\omega}+\Gamma_{\mathbf{u}}+\Delta \mathbf{M}+\mathbf{d}
\end{array}\right.
$$

In order to further develop our control scheme, the following lemmas and assumption are given. 
Assumption 1. It is supposed that lumped disturbances $d_{i}$ $(i=1,2,3)$ is continuous, which satisfy $\max \{\|\mathbf{d}\|,\|\dot{d}\|\} \leq \rho$ where $\rho$ is an unknown positive constant.

Lemma 1 (see [27]). A first-order sliding-mode differentiator is designed as

$$
\left\{\begin{array}{l}
\dot{\mu}_{1}=\varsigma, \\
\varsigma=\mu_{2}-b_{1}^{*} \cdot\left|\mu_{1}-F(t)\right|^{1 / 2} \cdot \operatorname{sign}\left(\mu_{1}-F(t)\right), \\
\dot{\mu}_{2}=-b_{2}^{*} \cdot \operatorname{sign}\left(\mu_{2}-\varsigma\right),
\end{array}\right.
$$

where $\mu_{1}$ and $\mu_{2}$ are the states of the differentiator (7), $\varsigma$ is the output of the differentiator, $b_{1}^{*}$ and $b_{2}^{*}$ are the positive constants, and $F(t)$ is a known function. Then, $\varsigma$ can approximate the differential term $\dot{F}(t)$ to any arbitrary accuracy if the initial deviations $\mu_{1}-F\left(t_{0}\right)$ and $\varsigma-\dot{F}\left(t_{0}\right)$ are bounded.

Lemma 2 (see [27]). Consider the system $\dot{x}=f(x, u)$. Suppose that there exist a continuous function $V(x)$ and scalars $\psi_{1}>0,0<\psi_{2}<1$, and $0<\psi_{3}<\infty$, such that

$$
\dot{V}(x) \leq-\psi_{1} \cdot V^{\psi_{2}}(x)+\psi_{3} \text {. }
$$

Then, this system's trajectory is practical finite-time stable.

2.2. Description of a Fuzzy Logic System. From [28], any continuous functions on a compact set can be approximated by the fuzzy logic system, with arbitrary accuracy. The fuzzy logic system is composed of four principal sections: an inference engine, a fuzzy rule, a fuzzifier, and a defuzzifier. Indeed, a set of 'If-Then' linguistic rules can describe the fuzzy logic system, i.e.,

$$
R^{(j)} \text { : If } x_{1} \text { is } A_{1}^{j} \text { and } \ldots \text { and } x_{n} \text { is } A_{n}^{j}, \quad \text { then } z \text { is } B^{j},
$$

where $j=1,2, \ldots, N ; x=\left(x_{1}, x_{2}, \ldots, x_{n}\right)^{T}$ and $z \in \mathbb{R}$ are the inputs and outputs of the fuzzy logic system, respectively; in addition, $A_{i}^{j}, i=1,2, \ldots, N$ and $B_{i}^{j}$ are characterized by the fuzzy membership functions $\mu_{A_{i}^{j}}\left(x_{i}\right)$ and $\mu_{B_{i}^{j}}(z)$, respectively. Based on the singleton fuzzifier, product inference, center-average defuzzifier, and Gaussian membership function, the output of the fuzzy logic system can be written as

$$
f(x)=\sum_{j=1}^{M} \frac{\bar{z}^{j}\left(\prod_{i=1}^{n} \mu_{A_{i}^{j}}\left(x_{i}\right)\right)}{\sum_{j=1}^{M}\left(\prod_{i=1}^{n} \mu_{A_{i}^{j}}\left(x_{i}\right)\right)}=\Phi \cdot \zeta(x),
$$

where $\bar{z}^{j} \in \mathbb{R}$ is the point at which $\mu_{B^{j}}(z)$ achieves the maximum value, $\Phi=\left[\bar{z}^{1}, \bar{z}^{2}, \ldots, \bar{z}^{M}\right]$ is an adjustable parameter vector, and $\zeta=\left[\zeta_{1}(x), \zeta_{2}(x), \ldots, \zeta_{n}(x)\right]^{T}$ is the fuzzy basis function vector; each element of $\zeta(x)$ is

$$
\zeta_{i}(x)=\sum_{j=1}^{M} \frac{\prod_{i=1}^{n} \mu_{A_{i}^{j}}\left(x_{i}\right)}{\sum_{j=1}^{M}\left(\prod_{i=1}^{n} \mu_{A_{i}^{j}}\left(x_{i}\right)\right)} .
$$

It should be pointed out that fuzzy logic system is a powerful function approximation tool, and it can effectively deal with uncertainty [29]. For a continuous nonlinear function $\mathbf{D}$, it can be approximated by

$$
\mathbf{D}=f(x)=\boldsymbol{\Phi} \cdot \boldsymbol{\zeta}^{T}(x)+\boldsymbol{v},
$$

where $\Phi=\left[\Phi_{1}, \Phi_{2}, \ldots, \Phi_{n}\right]^{T}$ is an optimal weight vector and $v=\left[v_{1}, v_{2}, \ldots, v_{n}\right]^{T}$ is the error vector of fuzzy approximation.

Assumption 2. The optimal weight and approximation error of FLS are bounded such that $\|\Phi\| \leq \Phi_{M}$ and $\|\nu\| \leq \nu_{M}$, where $\Phi_{M}$ and $v_{M}$ are the positive constants.

The aim of this paper is to construct a closed-loop system with the self-adaption compensation algorithm which guarantees that the commanded signal $\Theta_{\mathbf{d}}$ is tracked by the system output $\Theta$ in finite time in the presence of the lumped disturbance and input constraints.

\section{Control Strategy Design}

In this section, an output feedback attitude control strategy is proposed under the framework of the NTSMC method, which guarantees the boundedness of all the signals in the control loop. To achieve high-accuracy tracking control, a control input updating algorithm is further designed to avoid control input constraints and to handle lumped disturbances in the system. The stability of the closed-loop system can be proved at the same time. Now, the detailed contents are stated as follows.

First, we define the system tracking error $\mathbf{e}_{1} \in \mathbb{R}^{3}$ and its derivative $\mathbf{e}_{2} \in \mathbb{R}^{3}$ as

$$
\begin{aligned}
& \mathbf{e}_{1}=\left[e_{11}, e_{12}, e_{13}\right]^{T}=\boldsymbol{\Theta}-\boldsymbol{\Theta}_{\mathbf{d}}, \\
& \mathbf{e}_{2}=\dot{e}_{1}=\left[e_{21}, e_{22}, e_{23}\right]^{T}=\dot{\Theta}-\dot{\Theta}_{\mathbf{d}},
\end{aligned}
$$

where $\Theta_{\mathbf{d}}=\left[\Theta_{d 1}, \Theta_{d 2}, \Theta_{d 3}\right]^{T}$ represents the desired commanded signal. To ensure fast and accurate attitude tracking performance of system (1), an NTSM-type sliding surface [30] $\mathbf{S}_{\mathbf{a}} \in \mathbb{R}^{3}$ and an auxiliary variable $\mathbf{z} \in \mathbb{R}^{3}$ are defined as

$$
\mathbf{S}_{\mathbf{a}}=\left[S_{a 1}, S_{a 2}, S_{a 3}\right]^{T}=\mathbf{e}_{1}+c \cdot \mathbf{e}_{2}^{\kappa} \cdot \operatorname{sign}\left(\mathbf{e}_{2}\right), \quad 1<\kappa<2, c>0.5,
$$

$$
\begin{aligned}
\mathbf{z} & =\left[z_{1}, z_{2}, z_{3}\right]^{T}=\Gamma_{\mathbf{u}}-\Gamma_{\varphi}=\left[\begin{array}{l}
\Gamma_{\mathbf{u}}\left(u_{1}\right) \\
\Gamma_{\mathbf{u}}\left(u_{2}\right) \\
\Gamma_{\mathbf{u}}\left(u_{3}\right)
\end{array}\right]-\left[\begin{array}{l}
\Gamma_{\varphi}\left(\varphi_{1}\right) \\
\Gamma_{\varphi}\left(\varphi_{2}\right) \\
\Gamma_{\varphi}\left(\varphi_{3}\right)
\end{array}\right] \\
& =\underbrace{\left[\begin{array}{l}
u_{M} \cdot \tanh \left(\frac{u_{1}}{u_{M}}\right) \\
u_{M} \cdot \tanh \left(\frac{u_{2}}{u_{M}}\right)
\end{array}\right]}_{\Gamma_{\mathbf{u}}}-\underbrace{\left[\begin{array}{l}
\varphi_{M} \cdot \tanh \left(\frac{\varphi_{1}}{\varphi_{M}}\right) \\
u_{M} \cdot \tanh \left(\frac{\varphi_{2}}{\varphi_{M}}\right)
\end{array}\right],}_{\Gamma_{\varphi}} \underbrace{\left[\begin{array}{l}
u_{M} \\
\varphi_{M}
\end{array}\right]}_{\varphi_{M}}]
\end{aligned}
$$


where $\mathbf{e}_{2}^{\kappa} \operatorname{sign}\left(\mathbf{e}_{2}\right)=\left[\left|e_{21}\right|^{\kappa} \operatorname{sign}\left(e_{21}\right),\left|e_{22}\right|^{\kappa} \operatorname{sign}\left(e_{22}\right),\left|e_{23}\right|^{\kappa} \operatorname{sign}\right.$ $\left.\left(e_{23}\right)\right]^{T}, \kappa=(p / q)$ with odd integers $p$ and $q$, and in addition, $\varphi_{M}=u_{M} \cdot \tanh (1)-b>0$ ( $b$ is the design parameter satisfying $2 b \ll 1)$ and $\varphi_{i}(i=1,2,3)$ will be designed later. For analysis simplification, $\varphi \triangleq\left[\varphi_{1}, \varphi_{2}, \varphi_{3}\right]^{T}$ in which $\varphi$ will be given later.
Remark 1. By choosing $1<\kappa<2$ and the expression form of $S_{a i}(i=1,2,3)$, the singularity problem for $e_{2 i}=\dot{e}_{1 i}=0$ can be avoided. Moreover, function $S_{a i}=e_{1 i}+c \cdot\left|e_{2 i}\right|^{\kappa} \cdot \operatorname{sign}\left(e_{2 i}\right)$ is continuous and differentiable although the signum operator and the absolute value are involved [31].

With the combination of (6), (13), (14), and (16), the derivative of the terminal sliding surface $\mathbf{S}_{\mathbf{a}}$ is given as follows:

$$
\begin{aligned}
\dot{S}_{\mathbf{a}} & =\left[\dot{S}_{a 1}, \dot{S}_{a 2}, \dot{S}_{a 3}\right]^{T}=\mathbf{e}_{2}+\kappa c \cdot\left|\mathbf{e}_{2}\right|^{\kappa-1} \cdot \dot{e}_{2} \\
& =\mathbf{e}_{2}+\kappa c \cdot\left|\mathbf{e}_{2}\right|^{\kappa-1} \cdot\left[\ddot{\Theta}-\ddot{\Theta}_{\mathbf{d}}\right]=\mathbf{e}_{2}+\kappa c \cdot\left|\mathbf{e}_{2}\right|^{\kappa-1} \cdot\left[-\mathbf{J}^{-1} \cdot \mathbf{N} \cdot \boldsymbol{\omega}+\mathbf{J}^{-1} \cdot \mathbf{z}+\mathbf{J}^{-1} \boldsymbol{\varphi}+\mathbf{J}^{-1} \mathbf{d}-\mathbf{J}^{-1}\left(\boldsymbol{\varphi}-\Gamma_{\varphi}\right)+\mathbf{J}^{-1} \Delta \mathbf{M}-\ddot{\Theta}_{\mathbf{d}}\right]=\mathbf{e}_{2}+\kappa c \cdot\left|\mathbf{e}_{2}\right|^{\kappa-1} \cdot(\boldsymbol{\varphi}+\mathbf{D}),
\end{aligned}
$$

with $\quad\left|\mathbf{e}_{2}\right|^{\kappa-1}=\operatorname{diag}\left\{\left|e_{21}\right|^{\kappa-1},\left|e_{22}\right|^{\kappa-1},\left|e_{23}\right|^{\kappa-1}\right\} \geq 0 \quad$ and $\mathbf{D}=-\mathbf{J}^{-1} \mathbf{N} \omega+\mathbf{J}^{-1} \mathbf{d}+\mathbf{J}^{-1} \Delta \mathbf{M}-\ddot{\Theta}_{\mathbf{d}}+\mathbf{J}^{-1} \mathbf{z}+\mathbf{J}^{-1} \Gamma_{\varphi}-\varphi$. Note that the mixture term $\mathbf{D}$ is a nonlinear function including the external disturbances and parameter perturbations. To eliminate the adverse effect caused by the lumped disturbance $\mathbf{D}$ on the system, the fuzzy logic system represented by (10) is employed to learn the unknown nonlinear function D due to its universal approximation property, i.e.,

$$
\mathbf{D}=\boldsymbol{\Phi} \cdot \zeta^{T}+\boldsymbol{v}=\|\boldsymbol{\Phi}\| \cdot \boldsymbol{\Phi}^{*} \cdot \zeta^{T}+\boldsymbol{v},
$$

with $\Phi^{*}=(\Phi /\|\Phi\|)$. With the help of Young's inequality, the relationships (19) and (20) can be established:

$$
\begin{aligned}
& \mathbf{S}_{\mathbf{a}}^{T} \mathbf{e}_{2} \leq \frac{\left\|\mathbf{S}_{\mathbf{a}}^{T} \mathbf{e}_{2}\right\|_{2}^{2}}{2 a_{1}^{2}}+\frac{a_{1}^{2}}{2}, \\
& \kappa c \mathbf{S}_{\mathbf{a}}^{T}\left|\mathbf{e}_{2}\right|^{\kappa-1} \mathbf{D}=\kappa c \mathbf{S}_{\mathbf{a}}^{T}\left|\mathbf{e}_{2}\right|^{\kappa-1} \cdot\left(\|\boldsymbol{\Phi}\| \cdot \boldsymbol{\Phi}^{*} \cdot \boldsymbol{\zeta}+\boldsymbol{v}\right) \\
& \leq \frac{\kappa^{2} c^{2}\left\|\mathbf{S}_{\mathbf{a}}^{T} \mathbf{e}_{2}^{\kappa-1}\right\|_{2}^{2}\|\boldsymbol{\Phi}\|_{2}^{2}\|\zeta\|_{2}^{2}}{2 a_{2}^{2}} \\
& +\frac{a_{2}^{2}}{2}+\frac{\kappa^{2} c^{2}\left\|\mathbf{S}_{\mathbf{a}}^{T} \mathbf{e}_{2}^{\kappa-1}\right\|_{2}^{2}\|v\|_{2}^{2}}{2 a_{2}^{2}}+\frac{a_{2}^{2}}{2},
\end{aligned}
$$

where $\left\|\mathbf{S}_{\mathbf{a}}^{T} \mathbf{e}_{2}^{\kappa-1}\right\|_{2}^{2}=\left(S_{a 1} e_{21}^{\kappa-1}\right)^{2}+\left(S_{a 2} e_{22}^{\kappa-1}\right)^{2}+\left(S_{a 3} e_{23}^{\kappa-1}\right)^{2}$ and $\left\|\mathbf{S}_{\mathbf{a}}^{T} \mathbf{e}_{2}\right\|_{2}^{2}=\left(S_{a 1} e_{21}\right)^{2}+\left(S_{a 2} e_{22}\right)^{2}+\left(S_{a 3} e_{23}\right)^{2}$; moreover, since $0<\kappa-1=((p-q) / q)<1$, and based on the fact $e_{2 i}^{2(\kappa-1)} \leq e_{2 i}^{2}$ when $\left|e_{2 i}\right| \geq 1$ and $e_{2 i}^{2(\kappa-1)} \leq e_{2 i}^{\kappa-1}$ when $\left|e_{2 i}\right|<1$, we have

$$
\left\{\begin{array}{l}
\frac{\kappa^{2} c^{2}\left\|\mathbf{S}_{\mathbf{a}}^{T} \mathbf{e}_{2}^{\kappa-1}\right\|_{2}^{2}\|\boldsymbol{\Phi}\|_{2}^{2}\|\zeta\|_{2}^{2}}{2 a_{2}^{2}} \leq \frac{\kappa^{2} c^{2}\left\|\mathbf{S}_{\mathbf{a}}^{T} \mathbf{e}_{2}\right\|_{2}^{2}\|\boldsymbol{\Phi}\|_{2}^{2}\|\zeta\|_{2}^{2}}{2 a_{2}^{2}}+\frac{\kappa^{2} c^{2} \cdot \widehat{S}_{\mathbf{a}}^{T} \cdot \widehat{e}_{2}^{\kappa-1} \cdot\|\boldsymbol{\Phi}\|_{2}^{2}\|\zeta\|_{2}^{2}}{2 a_{2}^{2}}, \\
\frac{\kappa^{2} c^{2}\left\|\mathbf{S}_{\mathbf{a}}^{T} \mathbf{e}_{2}^{\kappa-1}\right\|_{2}^{2}\|\boldsymbol{v}\|_{2}^{2}}{2 a_{2}^{2}} \leq \frac{\kappa^{2} c^{2}\left\|\mathbf{S}_{\mathbf{a}}^{T} \mathbf{e}_{2}\right\|_{2}^{2}\|\boldsymbol{v}\|_{2}^{2}}{2 a_{2}^{2}}+\frac{\kappa^{2} c^{2} \cdot \widehat{S}_{\mathrm{a}}^{T} \cdot \widehat{e}_{2}^{\kappa-1}\|\boldsymbol{v}\|_{2}^{2}}{2 a_{2}^{2}},
\end{array}\right.
$$

where $\widehat{S}_{\mathbf{a}}=\left[S_{a 1}^{2}, S_{a 2}^{2}, S_{a 3}^{2}\right]^{T}$ and $\hat{e}_{2}^{\kappa-1}=\left[e_{21}^{\kappa-1}, e_{22}^{\kappa-1}, e_{23}^{\kappa-1}\right]^{T}$. From (21), (20) can be rewritten as

$$
\kappa c \mathbf{S}_{\mathbf{a}}^{T} \mathbf{e}_{2}^{\kappa-1} \mathbf{D} \leq \frac{\kappa^{2} c^{2}\left(\left\|\mathbf{S}_{\mathbf{a}}^{T} \mathbf{e}_{2}\right\|_{2}^{2}+\widehat{S}_{\mathbf{a}}^{T} \cdot \widehat{e}_{2}^{\kappa-1}\right)}{2 a_{2}^{2}} \cdot \boldsymbol{\eta}^{T} \psi+a_{2}^{2},
$$

where $\mathbf{e}_{2}^{\kappa-1}=\operatorname{diag}\left\{e_{21}^{\kappa-1}, e_{22}^{\kappa-1}, e_{23}^{\kappa-1}\right\}, \quad \eta=\left[\|\Phi\|^{2},\|\nu\|^{2}\right]^{T}$, and $\psi=\left[\|\zeta\|^{2}, 1\right]^{T}$. Next, a virtual control law $\varphi$ is designed as

$$
\boldsymbol{\varphi} \triangleq\left[\varphi_{1}, \varphi_{2}, \varphi_{3}\right]^{T}=-\frac{\kappa c}{2 a_{2}^{2}}\left(\mathbf{S}_{\mathbf{a}}+\mathbf{e}_{2}^{3-\kappa} \mathbf{S}_{\mathbf{a}}\right) \cdot\left(\widehat{\eta}^{T} \psi\right)-\mathbf{e}_{2}^{3-\kappa} \mathbf{S}_{\mathbf{a}}
$$

where $\mathbf{e}_{2}^{3-\kappa}=\operatorname{diag}\left\{e_{21}^{3-\kappa}, e_{22}^{3-\kappa}, e_{23}^{3-\kappa}\right\}$, and the related adaptation law is choosen as

$$
\dot{\hat{\eta}}=\gamma \cdot\left(\frac{\kappa^{2} c^{2}}{2 a_{2}^{2}} \cdot\left(\widehat{S}_{\mathbf{a}}^{T} \cdot-_{2}^{\kappa-1}+\left\|\mathbf{S}_{\mathbf{a}}^{T} \mathbf{e}_{2}\right\|_{2}^{2}\right) \cdot \psi-a_{2} \cdot \hat{\eta}\right), \quad \boldsymbol{\eta}(0)=0, \gamma>0 .
$$

In this study, $\widehat{\eta} \triangleq\left[\widehat{\eta}_{1}, \widehat{\eta}_{2}\right]^{T}$ is employed to estimate $\eta$, and the estimate error $\widetilde{\eta}$ denotes $\widetilde{\eta}=\eta-\widehat{\eta}$. It is worth noting that the time derivative of $\varphi$ (in (23)) is required for the subsequent attitude controller and stability analysis. It is not difficult to find that the derivative of the virtual law $\varphi$ can lead to the explosion problem of the complex term. In order to solve this problem, a first-order sliding-mode 
Mathematical Problems in Engineering

5

differentiator is introduced into the closed-loop system design to estimate each element of $\dot{\varphi}$ :

$$
\left\{\begin{array}{l}
\dot{\mu}_{1 i}=\varsigma_{i}=\mu_{2 i}-b_{1}^{*} \cdot\left|\mu_{1}-\varphi_{i}\right|^{1 / 2} \cdot \operatorname{sign}\left(\mu_{1}-\varphi_{i}\right), \\
\dot{\mu}_{2 i}=-b_{2}^{*} \cdot \operatorname{sign}\left(\mu_{2 i}-\varsigma_{i}\right), \\
i=1,2,3
\end{array}\right.
$$

where $\mu_{1 i}$ and $\mu_{2 i}$ are the states of the differentiator, $\varsigma_{i}$ is the output of the differentiator, and $b_{1}^{*}$ and $b_{2}^{*}$ are the positive design constants. According to (25) and Lemma 1, we obtain

$$
\dot{\varphi}=\varsigma+\varepsilon,
$$

where $\varsigma=\left[\varsigma_{1}, \varsigma_{2}, \varsigma_{3}\right]^{T}$ and $\varepsilon=\left[\varepsilon_{1}, \varepsilon_{2}, \varepsilon_{3}\right]^{T}$ is the estimation error vector of the introduced differentiator. According to Lemma 1 , we can get $\|\varepsilon\| \leq \delta$ with a positive constant $\delta$.

Noting that $\mathbf{S}_{\mathbf{a}}^{T} \mathbf{e}_{2}^{\kappa-1} \mathbf{S}_{\mathbf{a}}=\widehat{S}_{\mathbf{a}}^{T} \hat{e}_{2}^{\kappa-1}$ and $\mathbf{S}_{\mathbf{a}}^{T} \mathbf{e}_{2}^{\kappa-1} \mathbf{e}_{2}^{3-\kappa} \mathbf{S}_{\mathbf{a}}=$ $\left\|\mathbf{S}_{\mathbf{a}}^{T} \mathbf{e}_{2}\right\|_{2}^{2}$, we can get from (23) that

$$
\begin{gathered}
\kappa c \mathbf{S}_{\mathbf{a}}^{T} \mathbf{e}_{2}^{\kappa-1} \cdot \boldsymbol{\varphi} \leq-\frac{\kappa^{2} c^{2}}{2 a_{2}^{2}} \cdot \widehat{S}_{\mathbf{a}}^{T} \cdot \widehat{e}_{2}^{\kappa-1} \cdot \widehat{\eta}^{T} \boldsymbol{\psi} \\
-\frac{\kappa^{2} c^{2}}{2 a_{2}^{2}}\left\|\mathbf{S}_{\mathbf{a}}^{T} \mathbf{e}_{2}\right\|_{2}^{2} \cdot \widehat{\eta}^{T} \boldsymbol{\psi}-\kappa c\left\|\mathbf{S}_{\mathbf{a}}^{T} \mathbf{e}_{2}\right\|_{2}^{2} .
\end{gathered}
$$

Owing to $\widehat{\eta}^{T} \psi+\widetilde{\eta}^{T} \psi=\eta^{T} \psi$, we have

$$
\left\{\begin{array}{l}
-\frac{\kappa^{2} c^{2}}{2 a_{2}^{2}} \cdot\left\|\mathbf{S}_{\mathbf{a}}^{T} \mathbf{e}_{2}\right\|_{2}^{2} \cdot \widehat{\eta}^{T} \psi-\frac{\kappa^{2} c^{2}}{2 a_{2}^{2}}\left\|\mathbf{S}_{\mathbf{a}}^{T} \mathbf{e}_{2}\right\|_{2}^{2} \cdot \widetilde{\eta}^{T} \psi=-\frac{\kappa^{2} c^{2}}{2 a_{2}^{2}}\left\|\mathbf{S}_{\mathbf{a}}^{T} \mathbf{e}_{2}\right\|_{2}^{2} \cdot \boldsymbol{\eta}^{T} \psi \\
-\frac{\kappa^{2} c^{2}}{2 a_{2}^{2}} \cdot \widehat{S}_{\mathbf{a}}^{T} \widehat{e}_{2}^{\kappa-1} \cdot \widehat{\eta}^{T} \psi-\frac{\kappa^{2} c^{2}}{2 a_{2}^{2}} \cdot \widehat{S}_{\mathbf{a}}^{T} \widehat{e}_{2}^{\kappa-1} \cdot \widetilde{\eta}^{T} \psi=-\frac{\kappa^{2} c^{2}}{2 a_{2}^{2}} \cdot \widehat{S}_{\mathbf{a}}^{T} \widehat{e}_{2}^{\kappa-1} \cdot \boldsymbol{\eta}^{T} \psi
\end{array}\right.
$$

By using (17), (19), (22), (24), (27a), and (27b), the following relationship can be established:

$$
\begin{aligned}
& \mathbf{S}_{\mathbf{a}}^{T} \dot{S}_{\mathbf{a}}-\frac{1}{\gamma} \widetilde{\eta}^{T} \dot{\hat{\eta}} \leq \frac{1}{2 a_{1}^{2}} \cdot\left\|\mathbf{S}_{\mathbf{a}}^{T} \mathbf{e}_{2}\right\|_{2}^{2}+\frac{a_{1}^{2}}{2}-\frac{\kappa^{2} c^{2}}{2 a_{2}^{2}} \cdot \widehat{S}_{\mathbf{a}}^{T} \hat{e}_{2}^{\kappa-1} \cdot \widehat{\eta}^{T} \boldsymbol{\psi} \\
& -\frac{\kappa^{2} c^{2}}{2 a_{2}^{2}} \cdot\left\|\mathbf{S}_{\mathbf{a}}^{T} \mathbf{e}_{2}\right\|_{2}^{2} \cdot \hat{\eta}^{T} \psi-\kappa c \cdot\left\|\mathbf{S}_{\mathbf{a}}^{T} \mathbf{e}_{2}\right\|_{2}^{2}+a_{2}^{2} \\
& +\frac{\kappa^{2} c^{2}}{2 a_{2}^{2}}\left(\left\|\mathbf{s}_{\mathbf{a}}^{T} \mathbf{e}_{2}\right\|_{2}^{2}+\widehat{S}_{\mathbf{a}}^{T} \hat{e}_{2}^{\kappa-1}\right) \cdot \boldsymbol{\eta}^{T} \boldsymbol{\psi} \\
& -\frac{\kappa^{2} c^{2}}{2 a_{2}^{2}}\left(\widehat{S}_{\mathbf{a}}^{T} \hat{e}_{2}^{\kappa-1}+\left\|\mathbf{S}_{\mathbf{a}}^{T} \mathbf{e}_{2}\right\|_{2}^{2}\right) \cdot \tilde{\eta}^{T} \psi+a_{2} \tilde{\eta}^{T} \hat{\eta} \\
& \leq-\left(\kappa c-\left(2 a_{1}^{2}\right)^{-1}\right) \cdot\left\|\mathbf{S}_{\mathbf{a}}^{T} \mathbf{e}_{2}\right\|_{2}^{2}+0.5 a_{1}^{2}+a_{2}^{2}+a_{2} \tilde{\eta}^{T} \widehat{\eta} .
\end{aligned}
$$

To proceed, the dynamics of an auxiliary variable $\mathbf{z}$ in (16) can be calculated as

$$
\dot{z}=\frac{\partial \Gamma_{\mathbf{u}}}{\partial \mathbf{u}} \cdot \dot{u}-\frac{\partial \Gamma_{\varphi}}{\partial \varphi} \cdot \dot{\varphi}=\Gamma_{u} \cdot \dot{u}-\Gamma_{\varphi} \cdot(\varsigma+\varepsilon),
$$

where $\dot{u}=\left[\dot{u}_{1}, \dot{u}_{2}, \dot{u}_{3}\right]^{T}$,

$$
\begin{aligned}
& \Gamma_{\mathbf{u}}=\operatorname{diag}\left\{\Xi_{u 1}, \Xi_{u 2}, \Xi_{u 3}\right\}=\operatorname{diag}\left\{\frac{\partial \Gamma_{\mathbf{u}}\left(u_{1}\right)}{\partial u_{1}}, \frac{\partial \Gamma_{\mathbf{u}}\left(u_{2}\right)}{\partial u_{2}}, \frac{\partial \Gamma_{\mathbf{u}}\left(u_{3}\right)}{\partial u_{3}}\right\}=\frac{\partial \Gamma_{\mathbf{u}}}{\partial \mathbf{u}} \\
& =\left[\begin{array}{ccc}
\frac{4}{\left(e^{u_{1} / u_{M}}+e^{-u_{1} / u_{M}}\right)^{2}} & 0 & 0 \\
0 & \frac{4}{\left(e^{u_{2} / u_{M}}+e^{-u_{2} / u_{M}}\right)^{2}} & 0 \\
0 & 0 & \frac{4}{\left(e^{u_{3} / u_{M}}+e^{-u_{3} / u_{M}}\right)^{2}}
\end{array}\right], \\
& \Gamma_{\varphi}=\operatorname{diag}\left\{\Xi_{\varphi 1}, \Xi_{\varphi 2}, \Xi_{\varphi 3}\right\}=\operatorname{diag}\left\{\frac{\partial \Gamma_{\varphi}\left(\varphi_{1}\right)}{\partial \varphi_{1}}, \frac{\partial \Gamma_{\varphi}\left(\varphi_{2}\right)}{\partial \varphi_{2}}, \frac{\partial \Gamma_{\varphi}\left(\varphi_{3}\right)}{\partial \varphi_{3}}\right\}=\frac{\partial \Gamma_{\varphi}}{\partial \varphi} \\
& =\left[\begin{array}{ccc}
\frac{4}{\left(e^{\varphi_{1} / \varphi_{M}}+e^{-\varphi_{1} / \varphi_{M}}\right)^{2}} & 0 & 0 \\
0 & \frac{4}{\left(e^{\varphi_{2} / \varphi_{M}}+e^{-\varphi_{2} / \varphi_{M}}\right)^{2}} & 0 \\
0 & 0 & \frac{4}{\left(e^{\varphi_{2} / \varphi_{M}}+e^{-\varphi_{2} / \varphi_{M}}\right)^{2}}
\end{array}\right]
\end{aligned}
$$




$$
\begin{aligned}
= & \operatorname{diag}\left\{\frac{4}{\left(e^{\varphi_{1} /\left(u_{M} \cdot \tanh (1)-b\right)}+e^{-\left(\varphi_{1} /\left(u_{M} \cdot \tanh (1)-b\right)\right)}\right)^{2}}, \frac{4}{\left(e^{\varphi_{2} /\left(u_{M} \cdot \tanh (1)-b\right)}+e^{-\left(\varphi_{2} /\left(u_{M} \cdot \tanh (1)-b\right)\right)\left(\varphi_{2} /\left(u_{M} \cdot \tanh (1)-b\right)\right)}\right)^{2}},\right. \\
& \left.\frac{4}{\left(e^{\varphi_{3} /\left(u_{M} \cdot \tanh (1)-b\right)}+e^{-\left(\varphi_{3} /\left(u_{M} \cdot \tanh (1)-b\right)\right)\left(\varphi_{3} / u_{M} \cdot \tanh (1)-b\right)}\right)^{2}}\right\} .
\end{aligned}
$$

Now, the input update law $\dot{u}_{i}(i=1,2,3)$ is designed as

$$
\dot{u}_{i}= \begin{cases}\Xi_{u i}^{-1} \cdot\left(\sigma_{i}-\left(\frac{u_{i}^{2}}{2 u_{M}^{2}}+b\right) \cdot\left(1+\sigma_{i}^{2}\right)\right), & \text { if } u_{i} \geq u_{M} ; \\ \Xi_{u i}^{-1} \cdot \sigma_{i}, & \text { if }-u_{M}<u_{i}<u_{M} \\ \Xi_{u i}^{-1} \cdot\left(\sigma_{i}+\left(\frac{u_{i}^{2}}{2 u_{M}^{2}}+b\right) \cdot\left(1+\sigma_{i}^{2}\right)\right), & \text { if } u_{i} \leq-u_{M}\end{cases}
$$

where $u_{i}(0)=0$ and $\sigma \triangleq\left[\sigma_{1}, \sigma_{2}, \sigma_{3}\right]^{T}=-\left(\|\varsigma\|_{2}^{2} \mathbf{z} / 2\right)-c \mathbf{z}^{q / p}-$ $c \mathbf{z}-\left(c \mathbf{z}\left\|\mathbf{S}_{\mathbf{a}}\right\|^{(p+q) / p} /\|\mathbf{z}\|_{2}^{2}\right), \quad\left(\|\varsigma\|_{2}^{2} \triangleq \sum_{i=1}^{3} \varsigma_{i}^{2}, \quad \mathbf{z}^{q / p} \triangleq\left[z_{1}^{q / p}, z_{2}^{q / p}\right.\right.$, $\left.z_{3}^{q / p}\right]^{T},\left\|\mathbf{S}_{\mathbf{a}}\right\|^{(p+q) / p} \triangleq \sum_{i=1}^{3}\left|S_{a i}\right|^{(p+q) / p}$, and $\left.\|\mathbf{z}\|_{2}^{2} \triangleq \sum_{i=1}^{3} z_{i}^{2}\right)$. To facilitate the stability analysis, we focus our attention on the term $\mathbf{z}^{T} \dot{z}$ which satisfies the following relationship:

(1) If $u_{i} \geq u_{M}$, we obtain $z_{i}=\Gamma_{\mathbf{u}}\left(u_{i}\right)-\Gamma_{\varphi}\left(\varphi_{i}\right)>0$; according to the fact $\Gamma_{\mathbf{u}}\left(u_{i}\right) \geq \Gamma_{\mathbf{u}}\left(u_{M}\right)$ $=u_{M} \cdot \tanh (1)$ and $\left|\Gamma_{\varphi}\left(\varphi_{i}\right)\right| \leq \varphi_{M}=u_{M} \tanh (1)-b$, it yields $\sum_{i=1}^{3} z_{i}\left(u_{i}^{2} /\left(2 u_{M}^{2}\right)+b\right)\left(1+\sigma_{i}^{2}\right) \geq 0$.

Then, taking into account that

$$
\begin{aligned}
\left|\Xi_{\varphi_{i}}\right| & =\left|\frac{\partial \Gamma_{\varphi}\left(\varphi_{i}\right)}{\partial \varphi_{i}}\right| \\
& =\left|\frac{4}{\left(e^{\varphi_{i} /\left(u_{M} \cdot \tanh (1)-b\right)}+e^{-\left(\varphi_{i} /\left(u_{M} \cdot \tanh (1)-b\right)\right)}\right)^{2}}\right| \leq 1, \\
\mathbf{z}^{T} \Gamma_{\varphi}(\varsigma+\boldsymbol{\varepsilon}) \leq & 0.5 \cdot\left(\|\mathbf{z}\|_{2}^{2}\|\varsigma\|_{2}^{2}+\left\|\boldsymbol{\Gamma}_{\varphi}\right\|_{2}^{2}+\|\mathbf{z}\|_{2}^{2}+\delta \cdot\left\|\boldsymbol{\Gamma}_{\varphi}\right\|_{2}^{2}\right),
\end{aligned}
$$

this leads to

$$
\begin{aligned}
\mathbf{z}^{T} \dot{z}= & \sum_{i=1}^{3} \Xi_{u i} \cdot z_{i} \cdot \dot{u}_{i}-\mathbf{z}^{T} \boldsymbol{\Gamma}_{\varphi} \cdot(\varsigma+\boldsymbol{\varepsilon}) \\
= & \mathbf{z}^{T} \boldsymbol{\sigma}-\sum_{i=1}^{3} z_{i} \cdot\left(\frac{u_{i}^{2}}{2 u_{M}^{2}}+b\right) \cdot\left(1+\sigma_{i}^{2}\right)-\mathbf{z}^{T} \boldsymbol{\Gamma}_{\varphi}(\varsigma+\boldsymbol{\varepsilon}) \\
\leq & \mathbf{z}^{T}\left(-c \mathbf{z}^{q / p}-c \mathbf{z}-\frac{\|c\|_{2}^{2} \mathbf{z}}{2}\right)-c\left\|\mathbf{S}_{\mathbf{a}}\right\|^{(p+q) / p}+\frac{\|\mathbf{z}\|_{2}^{2}\|c\|_{2}^{2}}{2} \\
& +\frac{3 \cdot(1+\delta)}{2}+\frac{\|\mathbf{z}\|_{2}^{2}}{2} \\
= & -c \mathbf{z}^{(p+q) / p}-c\left\|\mathbf{S}_{\mathbf{a}}\right\|^{(p+q) / p}-\left(c-\frac{1}{2}\right)\|\mathbf{z}\|_{2}^{2}+\frac{3}{2}(1+\delta) .
\end{aligned}
$$

(2) If $\left|u_{i}\right|<u_{M}$, we can prove that

$$
\begin{aligned}
\mathbf{z}^{T} \dot{z}= & \sum_{i=1}^{3} \Xi_{u i} z_{i} \dot{u}_{i}-\mathbf{z}^{T} \cdot \boldsymbol{\Gamma}_{\varphi} \cdot(\varsigma+\boldsymbol{\varepsilon})=\mathbf{z}^{T} \boldsymbol{\Gamma}_{\mathbf{u}}-\mathbf{z}^{T} \boldsymbol{\Gamma}_{\varphi}(\varsigma+\boldsymbol{\varepsilon}) \\
\leq & \mathbf{z}^{T}\left(-c \mathbf{z}^{q / p}-c \mathbf{z}-\frac{\|\varsigma\|_{2}^{2} \mathbf{z}}{2}\right)-c\left\|\mathbf{S}_{\mathbf{a}}\right\|^{(p+q) / p}+\frac{\|\mathbf{z}\|_{2}^{2}\|\varsigma\|_{2}^{2}}{2} \\
& +\frac{3 \cdot(1+\delta)}{2}+\frac{\|\mathbf{z}\|_{2}^{2}}{2}=-c \mathbf{z}^{(p+q) / p}-c\left\|\mathbf{S}_{\mathbf{a}}\right\|^{(p+q) / p} \\
& -\left(c-\frac{1}{2}\right)\|\mathbf{z}\|_{2}^{2}+\frac{3(1+\delta)}{2} .
\end{aligned}
$$

(3) If $u_{i} \leq-u_{M}$, it can be easily inferred that $\Gamma_{\mathbf{u}}\left(u_{i}\right) \leq \Gamma_{\mathbf{u}}\left(-u_{M}\right)=-u_{M} \cdot \tanh (1)$. Owing to $\left|\Gamma_{\varphi}\left(\varphi_{i}\right)\right| \leq \varphi_{M}=u_{M} \cdot \tanh (1)-b$, we further obtain $z_{i}=\Gamma_{\mathbf{u}}\left(u_{i}\right)-\Gamma_{\varphi}\left(\varphi_{i}\right) \leq 0$, and this ensures that $\sum_{i=1}^{3} z_{i} \cdot\left(u_{i}^{2} /\left(2 u_{M}^{2}\right)+b\right) \cdot\left(1+\sigma_{i}^{2}\right) \leq 0$. Therefore,

$$
\begin{aligned}
\mathbf{z}^{T} \dot{z}= & \sum_{i=1}^{3} \Xi_{u i} z_{i} \dot{u}_{i}-\mathbf{z}^{T} \boldsymbol{\Gamma}_{\varphi}(\varsigma+\boldsymbol{\varepsilon}) \\
= & \mathbf{z}^{T} \boldsymbol{\sigma}+\sum_{i=1}^{3} z_{i} \cdot\left(\frac{u_{i}^{2}}{2 u_{M}^{2}}+b\right) \cdot\left(1+\sigma_{i}^{2}\right)-\mathbf{z}^{T} \cdot \boldsymbol{\Gamma}_{\varphi} \cdot(\varsigma+\boldsymbol{\varepsilon}) \\
\leq & \mathbf{z}^{T}\left(-c \mathbf{z}^{q / p}-c \mathbf{z}-\frac{\|s\|_{2}^{2} \mathbf{z}}{2}\right)-c\left\|\mathbf{S}_{\mathbf{a}}\right\|^{(p+q) / p}+\frac{\|\mathbf{z}\|_{2}^{2}\|\varsigma\|_{2}^{2}}{2} \\
& +\frac{3 \cdot(1+\delta)}{2}+\frac{\|\mathbf{z}\|_{2}^{2}}{2} \\
= & -c \mathbf{z}^{(p+q) / p}-c\left\|\mathbf{S}_{\mathbf{a}}\right\|^{(p+q) / p}-\left(c-\frac{1}{2}\right)\|\mathbf{z}\|_{2}^{2}+\frac{3(1+\delta)}{2} .
\end{aligned}
$$

Noting that the design parameter $c$ satisfies $c>0.5$ and combining with the above analysis, we thus obtain

$$
\mathbf{z}^{T} \dot{z} \leq-c \cdot \mathbf{z}^{(p+q) / p}-c \cdot\left\|\mathbf{S}_{\mathbf{a}}\right\|^{(p+q) / p}+\frac{3 \cdot(1+\delta)}{2} .
$$

Remark 2. It is noteworthy that the input update law $\dot{u}_{i}$ is necessary for the stability of the closed-loop system. Moreover, it can prevent $u_{i}$ from exceeding the working 
range. From (31), when $u_{i}=u_{M} \cdot \sqrt{1-2 b}$ (design parameter $b \quad$ satisfies $\quad 2 b \ll 1)$, it means $\dot{u}_{i}=\Xi_{u i}^{-1}\left(\sigma_{i}-\left(u_{i}^{2} /\left(2 u_{M}^{2}\right)+b\right) \cdot\left(1+\sigma_{i}^{2}\right)\right) \leq 0$; therefore, this prevents $u_{i}$ from getting greater than $u_{M} \cdot \sqrt{1-2 b}$. When $u_{i}=-u_{M} \sqrt{1-2 b}, \quad$ then $\quad \dot{u}_{i}=\Xi_{u i}^{-1}\left(\sigma_{i}+\left(u_{i}^{2} /\left(2 u_{M}^{2}\right)\right.\right.$ $\left.+b) \cdot\left(1+\sigma_{i}^{2}\right)\right) \geq 0$, and it implies that this prevents $u_{i}$ from getting smaller than $u_{i}=-\sqrt{1-2 b} \cdot u_{M}$.

The following theorem established the criteria for selecting the controller parameters to warrant the bounded stability of the attitude tracking error $e_{i}(i=1,2,3)$.

Theorem 1. Consider system (5) with Assumptions 1-2, and if the controller parameters $\left(a_{1}, a_{2}, c, b\right.$, and $\left.\kappa\right)$ are chosen as follows:

$$
\left\{\begin{array}{l}
0<a_{1}, \\
a_{2}<1, \\
c>0.5, \\
\kappa \cdot c>\left(2 a_{1}^{2}\right)^{-1}, \\
2 b \ll 1,
\end{array}\right.
$$

then the control structure composed of the virtual control law $\varphi$ (in (23)) with parameter update (24) and input update law $\dot{u}_{i}$ (in (31)) can insure that all the signals of the designed closed-loop system are bounded and $e_{1 i}(i=1,2,3)$ converges to a small neighborhood of the equilibrium point in finite time.

Proof. Consider the Lyapunov candidate function as

$$
V(t)=\frac{\mathbf{S}_{\mathbf{a}}^{T} \mathbf{S}_{\mathbf{a}}}{2}+\frac{\mathbf{z}^{T} \mathbf{z}}{2}+\frac{\widetilde{\eta}^{T} \widetilde{\eta}}{2 \gamma} .
$$

Taking the time derivative of $V(t)$ and applying (28) and (36), the following inequality can be obtained based on $\widetilde{\eta}=\eta-\hat{\eta}$ :

$$
\begin{aligned}
\dot{V}(t)= & \mathbf{S}_{\mathbf{a}}^{T} \dot{\boldsymbol{S}}_{\mathbf{a}}+\mathbf{z}^{T} \dot{z}-\gamma^{-1} \widetilde{\eta}^{T} \dot{\hat{\eta}} \\
\leq & -c \cdot \mathbf{z}^{(p+q) / p}-c \cdot\left\|\mathbf{S}_{\mathbf{a}}\right\|^{(p+q) / p}+a_{2} \widetilde{\eta}^{T} \widehat{\eta}+\frac{a_{1}^{2}}{2}+a_{2}^{2} \\
& +\frac{3}{2}(1+\delta) .
\end{aligned}
$$

By using Young's inequality, we can obtain that

$$
\begin{aligned}
a_{2} \widetilde{\eta}^{T} \widehat{\eta} & =a_{2} \widetilde{\eta}^{T}(\boldsymbol{\eta}-\widetilde{\eta}) \\
& =-a_{2} \widetilde{\eta}^{T} \widetilde{\eta}+a_{2} \widetilde{\eta}^{T} \boldsymbol{\eta} \leq a_{2}\left(-\widetilde{\eta}^{T} \widetilde{\eta}+\frac{1}{2} \widetilde{\eta}^{T} \widetilde{\eta}+\frac{1}{2} \boldsymbol{\eta}^{T} \boldsymbol{\eta}\right) \\
& =-\frac{a_{2}}{2 \gamma} \widetilde{\eta}^{T} \widetilde{\eta}+\frac{a_{2} \boldsymbol{\eta}^{T} \boldsymbol{\eta}}{2}-\left(\frac{a_{2} \gamma^{2}}{2 \gamma} \widetilde{\eta}^{T} \widetilde{\eta}\right)^{(p+q) / 2 p}+\left(\frac{a_{2} \gamma}{2 \gamma} \widetilde{\eta}^{T} \widetilde{\eta}\right)^{(p+q) / 2 p} .
\end{aligned}
$$

For Theorem 1 proof, the two cases are required to be addressed in the analysis of (40).

(1) If $a_{2} \gamma \cdot \widetilde{\eta}^{T} \widetilde{\eta} /(2 \gamma)>1$, the following inequality is true:

$$
-\frac{a_{2} \gamma \cdot \widetilde{\eta}^{T} \widetilde{\eta}}{2 \gamma}+\frac{a_{2} \gamma \cdot \widetilde{\eta}^{T} \widetilde{\eta}^{(p+q) / 2 p}}{2 \gamma} \leq 0 .
$$

(2) If $a_{2} \gamma \cdot \widetilde{\eta}^{T} \widetilde{\eta} /(2 \gamma) \leq 1$, the following relationship can be established:

$$
-\frac{a_{2} \gamma \cdot \widetilde{\eta}^{T} \widetilde{\eta}}{2 \gamma}+\frac{a_{2} \gamma \cdot \widetilde{\eta}^{T} \widetilde{\eta}^{(q+p) / 2 p}}{2 \gamma} \leq 1 .
$$

By the combination of (41) and (42), we have

$$
a_{2} \widetilde{\eta}^{T} \widehat{\eta} \leq-\left(\frac{a_{2} b \cdot \widetilde{\eta}^{T} \widetilde{\eta}}{2 \gamma}\right)^{(p+q) / 2 p}+0.5 \cdot a_{2} \cdot\left\|\boldsymbol{\eta}^{T}\right\|_{2}^{2}+1 \text {. }
$$

Using (43) results in

$$
\begin{aligned}
\dot{V}(t) \leq & -c \cdot 2^{(q+p) / 2 p} \cdot\left(\frac{\mathbf{z}^{T} \mathbf{z}}{2}\right)^{(q+p) / 2 p} \\
& -c \cdot 2^{(q+p) / 2 p} \cdot\left(\frac{\mathbf{S}_{\mathbf{a}}^{T} \mathbf{S}_{\mathbf{a}}}{2}\right)^{(q+p) / 2 p}-\left(a_{2} \gamma\right)^{(q+p) / 2 p} \\
& \cdot\left(\frac{a_{2} \gamma \cdot \tilde{\eta}^{T} \widetilde{\eta}}{2 \gamma}\right)^{(q+p) / 2 p}+\Delta \leq-\Phi \cdot V^{(q+p) / 2 p}+\Delta,
\end{aligned}
$$

where $\quad \Phi=\min \left\{c \cdot 2^{(q+p) / 2 p},(c \cdot \gamma)^{(q+p) / 2 p}\right\} \quad$ and $\Delta=0.5 \cdot a_{1}^{2}+a_{2}^{2}+1.5 \cdot(1+\delta)+0.5 a_{2}^{2} \cdot\left\|\eta^{T}\right\|_{2}^{2}+1$. Using Lemma 2, the decrease in $V(t)$ can force the sliding-mode variable of the closed-loop system into $V^{(q+p) / 2 p} \leq \Delta /[(1-$ $\left.\left.\beta^{*}\right) \cdot \varpi\right]$ with $0<\beta^{*}<1$, which implies that the terminal sliding-mode variable is bounded in finite time as

$$
\lim _{t \longrightarrow T_{f 1}} \mathbf{S}_{\mathbf{a}}(t) \in\left(\left\|\mathbf{S}_{\mathbf{a}}\right\| \leq\left(\frac{\Delta}{\left(1-\beta^{*}\right) \emptyset} \cdot 2^{p / 2(p+q)}\right)^{p /(p+q)}\right) .
$$

In the expression of (45), the finite time $T_{f 1}$ satisfies that $T_{f 1} \leq\left(\left[V\left(x_{0}\right)\right]^{(p-q) / p} /\left[\bowtie \beta^{*} \cdot((p-q) / p)\right]\right)$ in which $V\left(x_{0}\right)$ is the initial value of $V$. To describe the boundedness of attitude tracking errors, we need to reconsider each element of the NTSS shown in (15):

$$
e_{1 i}+c \cdot\left|e_{2 i}\right|^{\kappa} \cdot \operatorname{sign}\left(e_{2 i}\right)=S_{a i}, \quad i=1,2,3,
$$

where $\left|S_{a i}\right| \leq\left(\left(\Delta /\left(1-\beta^{*}\right) \emptyset\right) \cdot 2^{p / 2(p+q)}\right)^{p /(p+q)} \triangleq \xi$. Then, (46) can be rearranged as

$$
e_{1 i}+\bar{c} \cdot\left|e_{2 i}\right|^{\kappa} \cdot \operatorname{sign}\left(e_{2 i}\right)=0 \text {, }
$$

where $\bar{c} \triangleq c-S_{a i} \cdot\left|e_{2 i}\right|^{-\kappa} \cdot \operatorname{sign}\left(e_{2 i}\right)$. From (45) and (47), we consider the following two cases:

(1) If $\bar{c} \leq 0$, then it has been in the region $c\left|e_{2 i}\right|^{\kappa} \leq \xi$, and it is easy to obtain that $\left|e_{1 i}\right|$ converges to the region $\left|e_{1 i}\right|=\left|S_{a i}-c\right| \cdot\left|e_{2 i}\right|^{\kappa} \cdot \operatorname{sign}\left(e_{2 i}\right) \mid \leq 2 \xi$ in a finite time $T_{f 1}$ 
(2) If $\bar{c}>0$, (47) can be regarded as the sliding mode, and according to [31], it can be shown that the time required for $e_{1 i}$ along the nonsingular terminal sliding surface to reach 0 is $T_{f 2} \leq\left(\left(0.5 \cdot e_{1 i}^{2}\left(T_{f 1}\right)\right)^{1-\bar{\kappa}} /\left[\alpha^{*}(1-\bar{\kappa})\right]\right) \quad$ with $\bar{\kappa}=0.5\left(1+\kappa^{-1}\right)$ and $\alpha^{*}=2^{\bar{\kappa}} \cdot(1 / \bar{c})^{1 / \kappa}$; therefore, the convergence time of the tracking error $e_{1 i}$ is $T_{f}=T_{f 1}+T_{f 2}$

According to the above analysis, the stability of the designed closed-loop system is ensured through the sense of Lyapunov and the finite-time reachability is obtained, and the total convergence time of the closed-loop system is $T_{f} \leq T_{f 1}+T_{f 2}$.

Remark 3. Note that the convergence accuracy of the tracking error depends on the size of parameters $\Phi$ and $\Delta$. The smaller the desired $e_{1 i}(i=1,2,3)$, the larger the required parameters $1 / a_{1}, 1 / a_{2}, \kappa, c$, and $\gamma$. In practical application, such control parameters can be determined by using the trial-and-error method under condition (37) until a good performance is obtained.

Remark 4. From the point of view of improving the robust capability of the closed-loop system, the information of lumped disturbance should be integrated into the control scheme design accordingly. In the design of the closed-loop system, the fuzzy logic system is employed to obtain the information of the nonlinear mixture term $\mathbf{D}$. And, $\|\Phi\|$ is used in the fuzzy logic system estimation, as can be observed from $\eta=\left[\|\Phi\|^{2},\|\nu\|^{2}\right]^{T}$. So, the quantity of design update laws (24) can be completely independent from the amount of fuzzy logic rules.

Remark 5. In order to use the fuzzy approximation, the approximated function needs to be kept in a compact set during the operation process of the closed-loop system. For the proposed control strategy, such a condition is naturally fulfilled by the physical limitation of the quadrotor, and not only system states can be guaranteed to be bounded, but also all signals of the closed-loop system are confined in a compact set during the entire control process. The result is that the fuzzy logic system and fuzzy approximation can be reasonably used in the closed-loop system and perform its functions as approximation and learning for the lumped nonlinear function.

Remark 6. Compared with the input-saturation approximation approaches [32, 33], the proposed closed-loop control system utilizes the self-adjustment mechanism to prevent the design control command $u_{i}$ from exceeding $u_{M}$, which is adaptively compensated for the whole control process.

\section{Simulation Studies}

4.1. Parameter Setting. In order to validate the theoretical result, a simulation study is performed in this section (based on MATLAB/Simulink environment). The inertial matrix J and the Coriolis term $\mathbf{N}$ are given as follows:

$$
\begin{aligned}
\mathbf{J} & =\left[\begin{array}{lll}
J_{11} & J_{12} & J_{13} \\
J_{21} & J_{22} & J_{23} \\
J_{31} & J_{32} & J_{33}
\end{array}\right], \\
\mathbf{N} & =\left[\begin{array}{lll}
N_{11} & N_{12} & N_{13} \\
N_{21} & N_{22} & N_{23} \\
N_{31} & N_{32} & N_{33}
\end{array}\right],
\end{aligned}
$$

with $J_{11}=I_{1} \sin ^{2}(\beta)+I_{2} \cos ^{2}(\beta) \sin ^{2}(\alpha)+I_{3} \cos ^{2}(\beta) \cos ^{2}(\alpha)$, $J_{12}=\cos (\beta) \cos (\alpha) \sin (\alpha)\left(I_{2}-I_{3}\right), J_{13}=-I_{1} \sin (\beta), J_{21}=\cos$ $(\beta) \cos (\alpha) \sin (\alpha)\left(I_{2}-I_{3}\right), J_{22}=I_{2} \cos ^{2}(\alpha)+I_{3} \sin ^{2}(\alpha), J_{23}=0$, $J_{31}=-I_{1} \sin (\beta), J_{32}=0, J_{33}=I_{1}, N_{11}=\left(\left(I_{1} \beta \sin 2 \beta+I_{2}(-\dot{\beta}\right.\right.$ $\left.\sin 2 \beta \sin ^{2} \sigma+\dot{\sigma} \cos ^{2} \beta \sin 2 \sigma\right)-I_{3}\left(\beta \sin 2 \beta \cos ^{2} \sigma+\dot{\sigma} \cos ^{2} \beta\right.$ $\sin 2 \sigma)) / 2), \quad N_{12}=\left(I_{1} \dot{\alpha} \sin 2 \beta / 2\right)-I_{2}((\dot{\beta} \sin \beta \sin 2 \sigma / 2)-\dot{\sigma}$ $\left.\cos \beta \cos 2 \sigma+\left(\dot{\alpha} \sin 2 \beta \sin ^{2} \sigma / 2\right)\right)+I_{3}\left(-\dot{\alpha}\left(\sin 2 \beta \cos ^{2} \sigma / 2\right)\right.$ $-\dot{\sigma} \cos \beta \cos 2 \sigma+(\dot{\beta} \sin \beta \sin 2 \sigma / 2)), N_{13}=-I_{1} \dot{\beta} \cos \beta+\left(I_{2}-\right.$ $\left.I_{1}\right)\left(\dot{\alpha} \cos ^{2} \beta \sin 2 \sigma / 2\right), N_{21}=\left(-I_{1} \dot{\alpha} \sin 2 \beta / 2\right)+\left(I_{2} \dot{\alpha} \sin 2 \beta\right.$. $\left.\sin ^{2} \sigma / 2\right)+\left(I_{3} \dot{\alpha} \sin 2 \beta \quad \cos ^{2} \sigma / 2\right), \quad N_{22}=\left(\dot{\sigma} \sin 2 \sigma\left(I_{2}-\right.\right.$ $\left.\left.I_{3}\right) / 2\right), \quad N_{23}=I_{1} \dot{\alpha} \cos \beta+I_{2}(-(\dot{\beta} \sin 2 \sigma / 2)+\dot{\alpha} \cos \beta \cos 2 \sigma)+$ $I_{3}((\dot{\beta} \sin 2 \sigma / 2)-\dot{\alpha} \cos \beta \cos 2 \sigma), \quad N_{31}=\left(\dot{\alpha} \cos ^{2} \beta(\sin 2 \sigma)\right.$ $\left.\left(I_{3}-I_{2}\right) / 2\right), \quad N_{32}=-I_{1} \dot{\alpha} \cos \beta+I_{2}((\dot{\beta} \sin 2 \sigma / 2)-\dot{\alpha} \cos \beta$ $\cos 2 \sigma)$, and $N_{33}=0$, where $I_{1}=I_{2}=4.2 \times 10^{-3}$ $\left(\mathrm{N} \cdot \mathrm{m} / \mathrm{rad} / \mathrm{s}^{2}\right)$ and $I_{3}=8.5 \times 10^{-3}\left(\mathrm{~N} \cdot \mathrm{m} / \mathrm{rad} / \mathrm{s}^{2}\right)$.

Meanwhile, the disturbance $\mathbf{d}$ is selected as

$$
\mathbf{d}= \begin{cases}0.5 \cdot \mathbf{d}^{*}, & \text { for } 0 \leq t<6 \mathrm{~s} \\ 2.5 \cdot \mathbf{d}^{*}, & \text { for } 6 s \leq t<12 \mathrm{~s}\end{cases}
$$

where

$$
\mathbf{d}^{*}=0.01 \cdot\left[\begin{array}{c}
\sin \left(\frac{\pi t}{125}\right)+\sin \left(\frac{\pi t}{250}\right) \\
\sin \left(\frac{\pi t}{125}\right)+\cos \left(\frac{\pi t}{250}\right) \\
\cos \left(\frac{\pi t}{125}\right)+\sin \left(\frac{\pi t}{250}\right)
\end{array}\right] .
$$

The initial values of the quadrotor system are $\alpha(0)=-0.2 \mathrm{rad}, \quad \beta(0)=0.5 \mathrm{rad}, \quad \sigma(0)=1.2 \mathrm{rad}, \quad$ and $\dot{\Theta}=0.01 \mathrm{rad} / \mathrm{s}$. The control authority is assumed to be $u_{M}=0.4$, and the attitude angle commands are set to be

$$
\boldsymbol{\Theta}_{\mathbf{d}}=\left[\begin{array}{l}
\alpha_{d} \\
\beta_{d} \\
\sigma_{d}
\end{array}\right]=\left[\begin{array}{c}
0.8 \sin (t) \sin (0.4 t) \mathrm{rad} \\
0.6 \cos (0.5 t)(1-\cos (0.7 t)) \mathrm{rad} \\
0.6 \cos (0.7 t)(1-\cos (0.45 t)) \mathrm{rad}
\end{array}\right] .
$$

The parameters for the controller are chosen as follows: $p=5, q=3, a_{1}=a_{2}=0.05, c=2, b=0.001$, and $\gamma=5$. The differentiator parameters are selected as $b_{1}^{*}=b_{2}^{*}=5$; moreover, the fuzzy membership functions are given as 


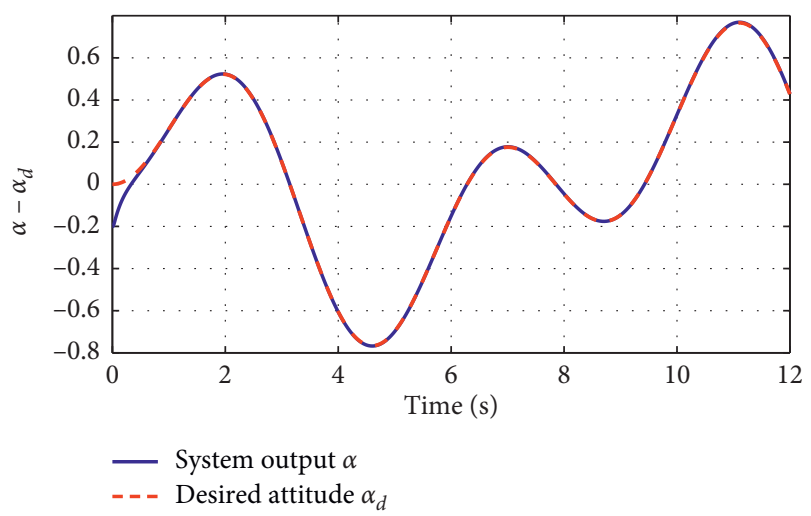

FIgUre 1: Attitude tracking $\alpha-\alpha_{d}$.

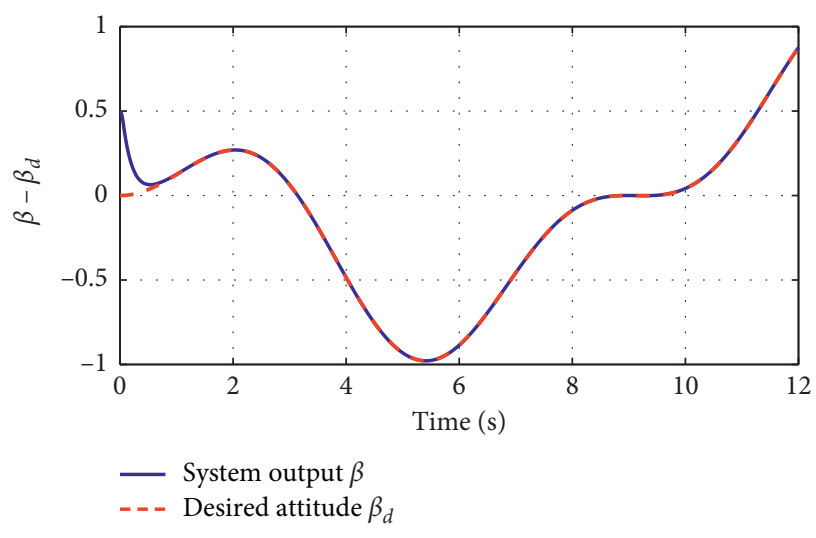

Figure 2: Attitude tracking $\beta-\beta_{d}$.

$$
\begin{aligned}
& \mu_{A_{i}^{1}}\left(x_{i}\right)=\exp \left[-\left(\frac{x_{i}+(\pi / 3)}{(\pi / 18)}\right)^{2}\right], \\
& \mu_{A_{i}^{2}}\left(x_{i}\right)=\exp \left[-\left(\frac{x_{i}+(\pi / 6)}{(\pi / 18)}\right)^{2}\right], \\
& \mu_{A_{i}^{3}}\left(x_{i}\right)=\exp \left[-\left(\frac{x_{i}}{(\pi / 18)}\right)^{2}\right], \\
& \mu_{A_{i}^{4}}\left(x_{i}\right)=\exp \left[-\left(\frac{x_{i}-(\pi / 6)}{(\pi / 18)}\right)^{2}\right], \\
& \mu_{A_{i}^{5}}\left(x_{i}\right)=\exp \left[-\left(\frac{x_{i}-(\pi / 3)}{(\pi / 18)}\right)^{2}\right] .
\end{aligned}
$$

4.2. Result Analysis. The attitude tracking response result is exhibited in Figures 1-3. Obviously, we can see that the command signals can be tracked by our control strategy with consideration of external disturbances and control input constraints. As can be seen from Figures 4 and 5, the proposed method achieves the goal of attitude control with high accuracy and fast convergence speed for attitude

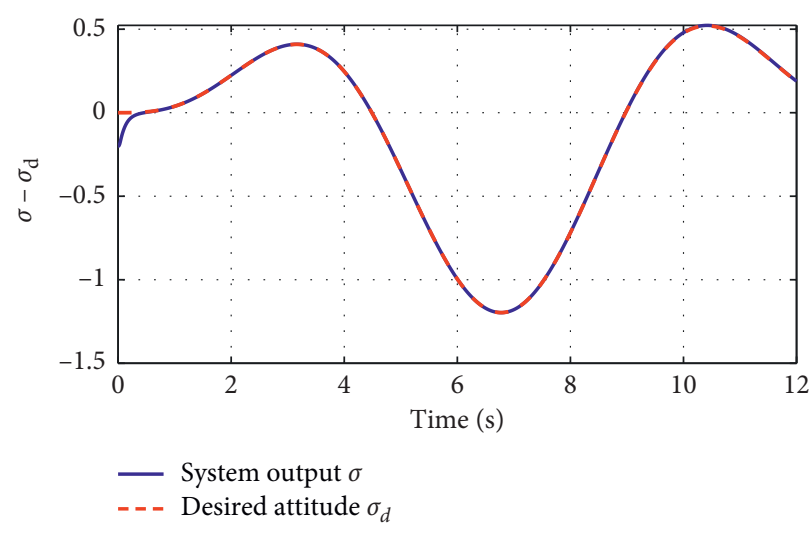

Figure 3: Attitude tracking $\sigma-\sigma_{d}$.

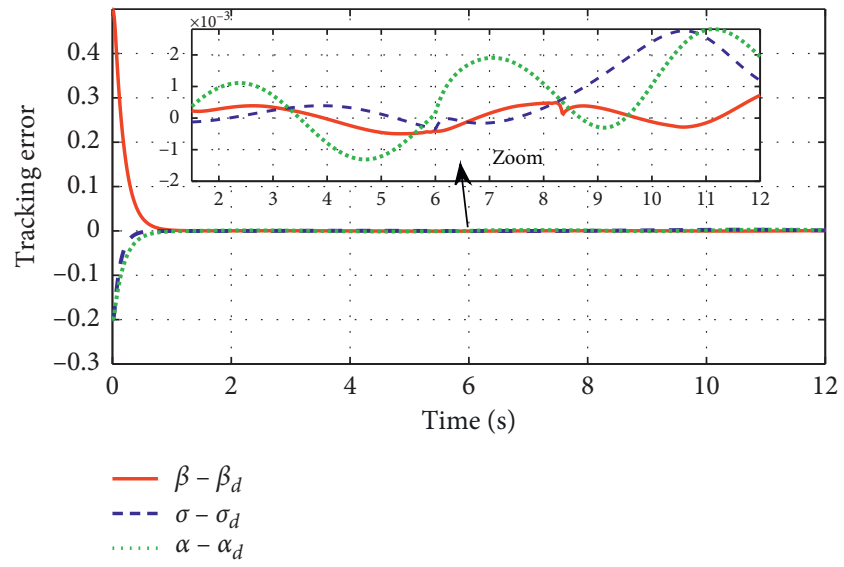

Figure 4: Attitude tracking error.

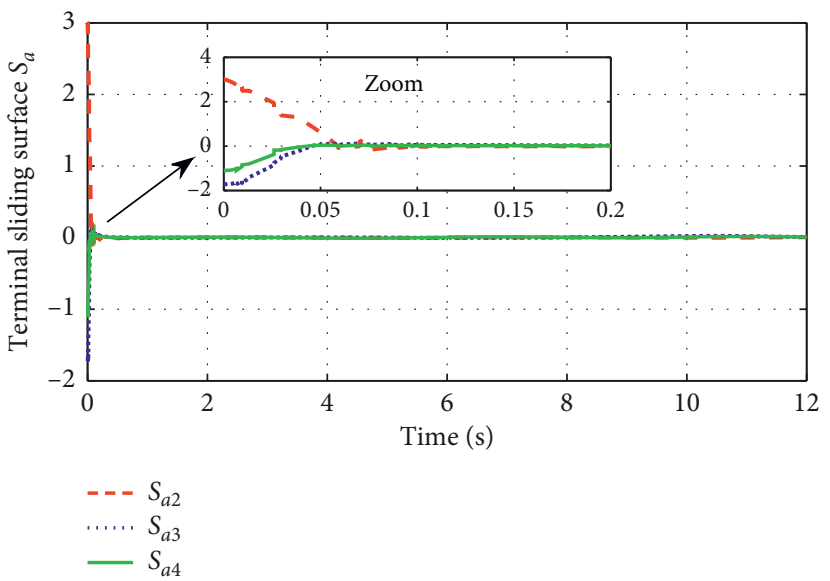

Figure 5: Dynamics of the terminal sliding surface.

tracking error trajectories $e_{1 i}(i=1,2,3)$. This is mainly because the proposed finite-time control scheme using the self-adaptation algorithm is capable of eliminating quickly the adverse influence caused by system uncertainty and external disturbances. During the increased disturbances $(t \geq 15 \mathrm{~s})$, the control performance based on the proposed 


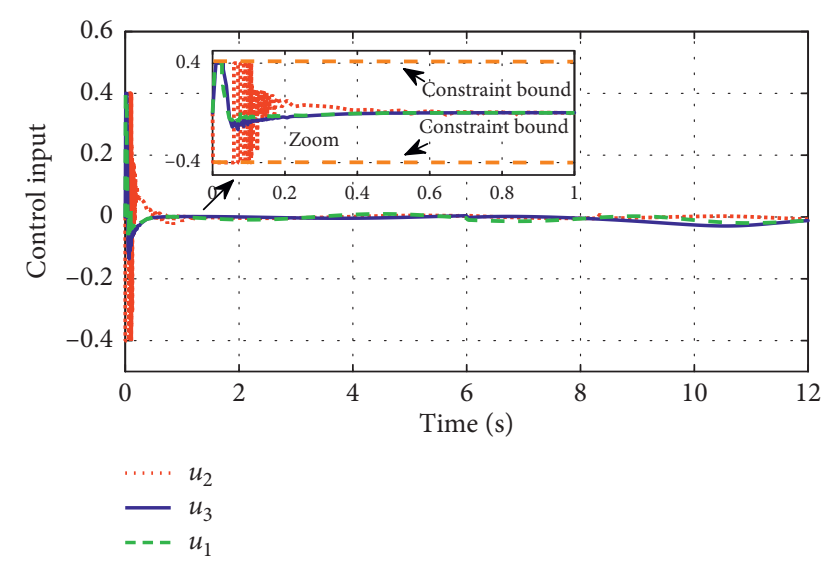

Figure 6: Control input.

control method is not obviously reduced because its selfadaption algorithm exerts accommodation process such that it can restore control performance as much as possible and further avoid the unstability of the closed-loop system with consideration of the increased external disturbances and control input constraints. The control input is as illustrated in Figure 6. Based on the above result and analysis, the effectiveness of the designed method can be validated.

\section{Conclusion}

In this study, a new model-free compensation control strategy using the finite-time technique is developed for attitude control of the quadrotor with adverse factors. The closed-loop system and the saturation suppression method are designed based on the NTSMC structure and the input parameter updating online strategy, respectively. A rigorous stability analysis and proof of the designed closed-loop system are combined with nonsingular terminal slidingmode theory and Lyapunov theory. The numerical simulation on the attitude control of the quadrotor is performed to validate the developed method.

\section{Data Availability}

The raw/processed data required to reproduce these findings cannot be shared at this time as the data also form a part of an ongoing study.

\section{Conflicts of Interest}

The authors declare that they have no conflicts of interest.

\section{References}

[1] H. Liu, J. Xi, and Y. Zhong, "Robust attitude stabilization for nonlinear quadrotor systems with uncertainties and delays," IEEE Transactions on Industrial Electronics, vol. 64, no. 7, pp. 5585-5594, 2017.

[2] Z. Zuo and S. Mallikarjunan, " $L 1$ adaptive backstepping for robust trajectory tracking of UAVs," IEEE Transactions on Industrial Electronics, vol. 64, no. 4, pp. 2944-2954, 2017.
[3] B. Tian, J. Cui, H. Lu, Z. Zuo, and Q. Zong, "Adaptive finitetime attitude tracking of quadrotors with experiments and comparisons," IEEE Transactions on Industrial Electronics, vol. 66, no. 12, pp. 9428-9438, 2019.

[4] Z. Y. Jia, J. Q. Yu, Y. S. Mei, Y. B. Chen, Y. Shen, and X. Ai, "Integral backstepping sliding mode control for quadrotor helicopter under external uncertain disturbances," Aerospace Science and Technology, vol. 68, pp. 299-307, 2017.

[5] K. Djamel, M. Abdellah, and A. Benallegue, "Attitude optimal backstepping controller based quaternion for a UAV," Mathematical Problems in Engineering, vol. 2016, Article ID 8573235, 11 pages, 2016.

[6] Z. Song and K. Sun, "Adaptive compensation control for attitude adjustment of quad-rotor unmanned aerial vehicle," ISA Transactions, vol. 69, pp. 242-255, 2017.

[7] Z. Song, S. Ling, and K. Sun, “Adaptive fault tolerant attitude tracking control for miniature rotorcrafts under actuator saturation," Aerospace Science and Technology, vol. 69, pp. 27-38, 2017.

[8] Z. K. Song and K. B. Sun, "Attitude tracking control of a quadrotor with partial loss of rotation effectiveness," Asian Journal of Control, vol. 19, pp. 1812-1821, 2017.

[9] Y. Yang and Y. Yan, "Attitude regulation for unmanned quadrotors using adaptive fuzzy gain-scheduling sliding mode control," Aerospace Science and Technology, vol. 54, pp. 208-217, 2016.

[10] H. Wang, X. Ye, Y. Tian, G. Zheng, and N. Christov, "Modelfree-based terminal SMC of quadrotor attitude and position," IEEE Transactions on Aerospace and Electronic Systems, vol. 52, no. 5, pp. 2519-2528, 2016.

[11] Z. K. Song, K. B. Sun, and S. Ling, "Stabilization and synchronization for a mechanical system via adaptive sliding mode control," ISA Transactions, vol. 68, pp. 353-366, 2017.

[12] H. B. Wang, Z. Li, H. Y. Xiong, and X. H. Nian, "Robust $H_{\infty}$ attitude tracking control of a quadrotor $\mathrm{UAV}$ on $\mathrm{SO}(3)$ via variation-based linearization and interval matrix approach," ISA Transactions, vol. 87, pp. 10-16, 2019.

[13] H. R. Jiang, Y. Q. Xia, R. Hu, D. L. Ma, and C. X. Hao, “A feedback linearization and saturated control structure for quadrotor UAV," in Proceedings of the 2019 Chinese Control Conference (CCC), Guangzhou, China, 2019.

[14] F.-J. Lin, S.-Y. Lee, and P.-H. Chou, "Intelligent nonsingular terminal sliding mode control using MIMO Elman neural network For Piezo-Flexural nanopositioning stage," IEEE Transactions on Ultrasonics, Ferroelectrics and Frequency Control, vol. 59, no. 12, pp. 2716-2730, 2012.

[15] Q. Xu, "Piezoelectric nanopositioning control using secondorder discrete-time terminal sliding mode strategy," IEEE Transactions on Industrial Electronics, vol. 62, no. 12, pp. 7738-7748, 2015.

[16] Y. Yang, "A time-specified nonsingular terminal sliding mode control approach for trajectory tracking of robotic airships," Nonlinear Dynamics, vol. 92, no. 3, pp. 1359-1367, 2018.

[17] M. L. Jin, J. Lee, and K. K. Ahn, "Continuous nonsingular terminal sliding mode control of shape memory alloy actuators using time delay estimation," IEEE/ASME Transactions on Mechatronics, vol. 20, no. 2, pp. 899-909, 2014.

[18] M. Kahouadji, M. R. Mokhtari, A. C. Braham, and B. Cherki, "Real-time attitude control of 3 DOF quadrotor UAV using modified super twisting algorithm," Journal of the Franklin Institute, vol. 357, no. 5, pp. 2681-2695, 2019.

[19] J. Zhou, Y. Y. Cheng, H. B. Du, D. Wu, M. Zhu, and X. Z. Lin, "Active finite-time disturbance rejection control for attitude 
tracking of quad-rotor under input saturation," Journal of the Franklin Institute, vol. 21, 2019.

[20] M. Labbadi and M. Cherkaoui, "Robust adaptive nonsingular fast terminal sliding-mode tracking control for an uncertain quadrotor UAV subjected to disturbances," ISA Transactions, vol. 99, pp. 290-304, 2019.

[21] W. Q. Gong, B. Li, Y. S. Yang, H. Y. Ban, and B. Xiao, "Fixedtime integral-type sliding mode control for the quadrotor UAV attitude stabilization under actuator failures," Aerospace Science and Technology, vol. 95, Article ID 105444, 2019.

[22] B. Xiao, Q. L. Hu, and Y. M. Zhang, "Adaptive sliding mode fault tolerant attitude tracking control for flexible spacecraft under actuator saturation," IEEE Transactions on Control Systems Technology, vol. 20, no. 6, pp. 1605-1612, 2012.

[23] Q. Hu, B. Xiao, and M. L. Friswell, "Robust fault-tolerant control for spacecraft attitude stabilisation subject to input saturation," IET Control Theory and Applications, vol. 5, no. 2, pp. 271-282, 2011.

[24] Q. Zhou, L. J. Wang, C. W. Wu, H. Y. Li, and H. P. Du, "Adaptive fuzzy control for Nonstrict-feedback systems with input saturation and output constraint," IEEE Transactions on Systems, Man, and Cybernetics: Systems, vol. 47, no. 1, pp. 1-12, 2017.

[25] Y. N. Yang, "Positioning control for stratospheric satellites subject to dynamics uncertainty and input constraints," Aerospace Science and Technology, vol. 86, pp. 534-541, 2019.

[26] W. He, Y. T. Dong, and C. Y. Sun, "Adaptive neural impedance control of a robotic manipulator with input saturation," IEEE Transactions on Systems, Man, and Cybernetics: Systems, vol. 46, no. 3, pp. 334-344, 2016.

[27] A. Levant, "Universal SISO sliding mode controllers with finite-time convergence," IEEE Transactions on Automatic Control, vol. 46, no. 9, pp. 1447-1451, 2001.

[28] Z. Zhu, Y. Q. Xia, and M. Y. Fu, "Attitude stabilization of rigid spacecraft with finite-time convergence," International Journal of Robust and Nonlinear Control, vol. 21, no. 6, pp. 686-702, 2011.

[29] L. -X. Wang, "Fuzzy systems are universal approximators," in Proceedings of the International Conference on Fuzzy Systems, pp. 1163-1170, San Diego, CA, USA, 1992.

[30] L.-X. Wang and J. M. Mendel, "Fuzzy basis function, universal approximation, and orthogonal least-squares learning," IEEE Transactions on Neural Networks, vol. 3, no. 5, pp. 807-814, 1992.

[31] Y. Feng, X. Yu, and Z. Man, "Non-singular terminal sliding mode control of rigid manipulators," Automatica, vol. 38, no. 12, pp. 2156-2167, 2002.

[32] S. H. Yu, X. H. Yu, B. Shirinzadeh, and Z. H. Man, "Continuous finite-time control for robotic manipulators with terminal sliding mode," Automatica, vol. 41, no. 11, pp. 1957-1964, 2005.

[33] S. Wang, W. H. Wang, and S. F. Xiong, "Impact angle constrained three-dimensional integrated guidance and control for STT missile in the presence of input saturation," ISA Transactions, vol. 64, pp. 151-160, 2016.

[34] Z. J. Liu, J. K. Liu, and W. He, "Modeling and vibration control of a flexible aerial refueling hose with variable lengths and input constraint," Automatica, vol. 77, pp. 302-310, 2017. 